\title{
An Efficient Regular Expression Pattern Matching Using Stride Finite Automata
}

\author{
Krishna Kishore Thota, R. JebersonRetna Raj
}

\begin{abstract}
Example coordinating assumes a key job in different parcel payload identification applications, for example, interruption location, which is utilized in distinguishing the malware content in system frameworks. Various calculations and instruments have been created to improve reality complexities of distinguishing regex principles and subsequently empower profound bundle review at line rate. In this paper, a novel quickening plan is introduced to determine speed and space wasteful aspects of the customary automata and the DFA called multi-walk Finite automata that confirms more than one byte that expands the general execution of not just design matching but additionally string coordinating.
\end{abstract}

Keywords: Malware Identification, Pattern Matching, Regular Expression

\section{INTRODUCTION}

As the data transfer rate of Internet traffic increases, the amount of suspicious information also increases and hence it is necessary to provide mechanisms to make the network data packets free from malicious attack. Network Intrusion Detection Systems have become the most efficient way for verifying the framework structures against these toxic strikes. It always screens the data bundles for malignant information and gives the alerts.

Significant Packet Inspection has become a key part in present day systems to perceive the strikes and diseases in framework traffic reliant on models set away in an attacker's database [1], whose structure can be either strings or regex

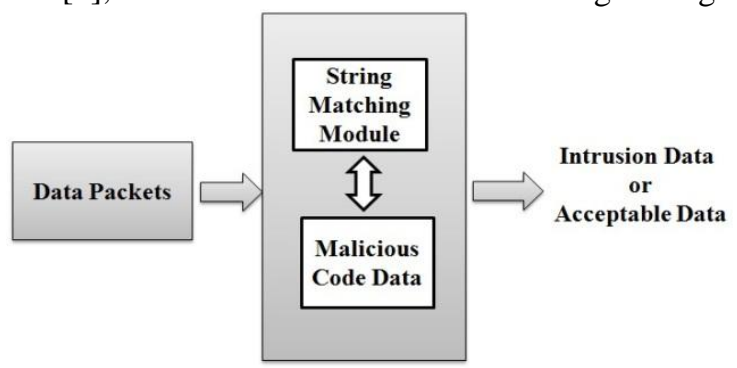

Fig.1. Intrusion Detection System Model

Stringcomparison has become the major component of Intrusion Detection Systems. In String matching, the input stream is compared with some pre-defined stored patterns to detect the malicious contents. These stored patterns are compared with the input stream. If the input data words as $\mathrm{W}=\mathrm{a}_{1} \mathrm{a}_{2} \ldots \mathrm{a}_{\mathrm{n}}$ and keyword $\mathrm{K}=\mathrm{k}_{1} \mathrm{k}_{2} \ldots \mathrm{k}_{\mathrm{n}}$, verify whether the keyword $\mathrm{K}$ exists as substring of data content $\mathrm{S}$. This endeavor is very direct yet it is used all around as frequently

Revised Manuscript Received on August 14, 2019.

Krishna Kishore Thota,Computer Science Engineering,Sathyabama Institute of Science and Technology,Chennai, Tamilnadu, India. Email thota.krishnakishore@gmail.com

Dr. R. JebersonRetna Raj, Associate Professor, Information Technology, Sathyabama Institute of Science and Technology, Chennai, Tamilnadu, India. Email: jebersonretnaraj.it@sathyabama.ac.in as conceivable in the areas, for instance, frameworks application. Particularly speedy computations are therefore fundamental for this execution.

To help continuously complex framework essentials, regex rules are replaced in the place of strings because of their over expressiveness and flexibility. However, structure organizing using standard verbalizations also encounters perfect speed and limit complexities, which limits its use in applications that prerequisites high taking care of speed or confined memory usage.

Organizing a novel model planning engine for both string and regex that achieves both reality profitability is a troublesome task, which can be practiced through Finite State Automata. It gives a compelling strategy to dealing with many string planning issues.

The most standard way to deal with execute regex is through Deterministic Finite Automaton (DFA) and Nondeterministic Finite Automaton (NFA). DFA has high computational speed in string processing but suffers from high storage complexity, whereas NFA is slower due to nondeterministic matching performance but consumes less memory [2]. Hence, neither of them is suitable for implementing high- speed and least memory consumption regex matching environments.

In this paper, a variable walk model sorting out motor is proposed that accomplish an unprecedented high string arranging execution with least memory use. Specifically, we propose walk Finite Automata (stride FA), that can channel different bytes reliably and when stood out from different tallies, it is relied upon to be repudiate the memory effect and information stream game-plan issues, and hereafter, requires the ideal memory.

The remaining part of this paper bargains with: section II manages the related research work done in this field and zone III introduces the evaluation background information of the work, partition IV manages the Multistoried Finite Automata, area $\mathrm{V}$ manages the proposed course of action, next the outcomes and the last section is the end part.

\section{RELATED WORK}

Model arranging is a noteworthy technique dependent on signature and utilized for obstruction hatred system. Most of the past work has been founded on rising the speed or perhaps lessening the memory cost of regex pattern matching [3]-[6]. These plans can be arranged into two portrayals: (1) single-byte walk isolating, and (2) multi-byte 
walk checking. Standard Automata near to a touch of their collections, including Hybrid [7], k-DFA [8], and XFA [19, [10], matches one byte and have a spot with the five star. Plans in the following sort take a gander at more bytes and accordingly, routinely give speedier arranging pace than those in the previous one.

Tune et al. [11], Lunteren [12] and Becchi and Crowley [13] accomplish memory decay by limiting advances in DFA, explicitly base on the mistaking issues related for standard articulation DFA.Yu et al. [5] joined fingerprints into DFAs dependent on avaricious method to not outflank maximum memory bound.

Tan and Sherwood [14] utilized different parallel DFA for isolating information characters. This controls the bits for the information photos of the DFA.Unnecessary off-chip gets to are killed in [15] utilizing Bloom Filters. TCAMs are utilized in [16] and [17] to rise the presentation of pattern arranging. They accomplish more speed with vague tradeoffs from chose in SRAM-based calculations. The application [18] utilizes multiprocessors on more information streams to scan for different models.

Clark and Schimmel [19] and Brodie et al. [20] have proposed equipment based frameworks that usemultibyte pictures for changes close by other optimizations. SidhuandPrasanna [21] made FPGA from NFA that outcomes in unnoticeable presentation. Karuppiah and Rajaram [22] beginning late made DFA from the standard expression, that diminishes all out states utilized outcomes the district efficiency.Sailesh et al [23] have given the model of D2FA, where the rehashed edges are disposed of and supplanted by the single default edge thatcomparesthe general DFA and the D2FA execution.

Regardless, most plans in multi-byte walk checking class experience the malevolent effects of two issues.

1. Exponential improvement in the memory fundamentals in light of genuine change in the overall transition numbers when the walk increments.

2. In multibit checking, every byte of the advancing toward data stream will influence the opportunity to be explored as the central character; this requires copy automata to convey the best sorting out outcomes.

The obligation of the present proposal will help in structure up an advantageous method for the model sorting out in the application over the system.

\section{FOUNDATION}

\section{A. Regular Expression}

Let $\sum$ be a letter set. The ordinary articulation (regex) over $\sum$ and its standard set can be characterized pursues:

- $\varphi$ is a regex that speaks to purge set.

- $\quad \epsilon$ is a regex that speaks to set containing invalid string.

- $\quad$ For each an in $\sum$, a will be a regex that speaks to the set $\{a\}$.

These are alluded as crude customary articulations.

- If $\mathrm{p}$ and $\mathrm{q}$ are customary articulations indicating the dialects $P$ and $Q$ separately, at that point $(p+q)$, (p.q) and (p)* means the sets P UQ, P.Q and $\mathrm{P} *$ individually.
- A string is a customary articulation iff it tends to be produced from crude ordinary articulations through limited use of the above guidelines.

- $\quad$ For example, $(0+1)^{*}$ means every one of the strings of 0 's and 1 's.

\section{B. Customary Sets}

An extraordinary class of words over $\mathrm{S}$ called standard sets is characterized as:

a) Every limited arrangement of words over $\mathrm{S}$, including invalid set.

b) If $\mathrm{P}$ and $\mathrm{Q}$ are standard sets over $\mathrm{S}$, at that point $\mathrm{P}$ UQ and P.Q are additionally customary.

c) If $\mathrm{R}$ is a standard set over $\mathrm{S}$, at that point so its conclusion $\mathrm{S}^{*}$.

d) A set is non-standard in the event that it isnot acquired by a limited number of utilizations of definitions from (a) to (c).

Hence, the class of regular sets are closed under Union, concatenation and Star operation.

For example, let $\sum=\{0,1\}$, then $\{01,10\}$ is a regular set.

\section{Nondeterministic Finite Automata}

In NFA, each state maps to multiple next states for any input symbol and all the states need not have mappings for all the input symbols. Such transitions are known as multivalued transitions.

$\delta$ is the mapping limit where $\delta: \mathrm{Q} \times \sum \rightarrow \mathrm{Q}$

$\mathrm{q} 0$ is the hidden state from where any data is taken care of $(\mathrm{q} 0 € \mathrm{Q})$.

Formal Definition of a NFA

A NFA can be addressed by a 5-tuple $\left(\mathrm{Q}, \sum, \delta, \mathrm{q} 0, \mathrm{~F}\right)$ where,

$\mathrm{Q}$ is a constrained game plan of states.

$\sum$ is ainput letters all together.

$\delta$ is the mappingn work where $\delta: \mathrm{Q} \times \sum \rightarrow 2 \mathrm{Q}$

$\mathrm{q} 0$ is the hidden state from where any data is readied (q0 $€ \mathrm{Q})$.

$\mathrm{F}$ is a ton of decisive state/states of $\mathrm{Q}(\mathrm{F} \square \mathrm{Q})$.

\section{Deterministic Finite Automata}

In DFA, no data picture causes to move more than one state for the given data picture.

Each and every state needs to eat up all the information pictures present in $\sum$.

\section{$D F A$}

Authoritatively described by a 5-tuple documentation $(\mathrm{Q}$, $\left.\sum, \delta, \mathrm{q} 0, \mathrm{~F}\right)$, where

$\mathrm{Q}$ is a restricted course of action of states.

$\sum$ is ainput letter set.

$\mathrm{F}$ is a great deal of convincing state, $\mathrm{F} \square \mathrm{Q}$.

These general methods differ in transitions.DFA allows only unique single valued transitions such that each state have only one next state for any input symbol, whereas NFA permits multi-valued transitions such that a state contain more than one next state for an input symbol. Unlike DFA, all states need not have mappings for all the input symbols. 
In NFA, the outcome is a subset of Q, where as in DFA, the outcome is an element of Q.

\section{MULTI-STRIDE DFA OVERVIEW\& RESULTS}

In this paper, we developed various walk model planning engine that have a fruitful string organizing execution with low memory usage. In particular, we propose walk restricted machine (Stride FA), that examines more than one data bytes in a steady progression.

Walk Finite Automaton (Stri FA), not at all like general restricted automata model, stimulates both string and standard verbalization matching. The normal constrained automata, check the entire data traffic to distinguish suspicious data, a StriFA can perceive malignant data by exploring simply inadequate input stream.

The data bundle is first changed into a short length entire number stream. Directly, differentiate this int byte stream and a variety of customary automata, called StriNFA or DFA, to recognize malicious substance in the given data.

Transforming the given input data into integer stride stream:

- Some particular characters known as tag are chosen.

- The distance from one tag to otheris known as stride length (SL).

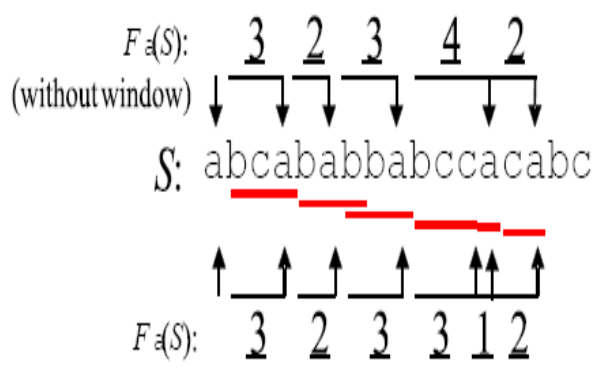

(with a window of size 3)

Fig.2. Use tag (an) and sliding window with size 3 to change data stream into Stride Length steam

\section{An Example of StrideFA}

Expect standard explanation be rule as ".*abba. $\{2\}$ caca"

$\mathrm{Fa}(*$ abba $)$ is $(1 / 2 / 3)+3$.

The great ways from starting an in "caca" depends upon two discretionary characters which can be both of the going with strings: [^a][^a], a[^a], [^a]a, or aa.

So possibly the entire number walk lengthstream of $\mathrm{Fa}(.\{2\}$ caca $)=$

$312([\wedge \mathrm{a}][\wedge \mathrm{a}]$ caca $)$

132 (a $[\wedge \mathrm{a}]$ caca)

222 ([^a] acaca)

1122 (aacaca)

$\mathrm{Fa}(*$ *abba. $\{2\}$ caca $)=\left(\begin{array}{l|l|l}1 & 2 & 3\end{array}\right)+3\left(\begin{array}{lll|lll|lll|l}3 & 1 & 2 & 1 & 3 & 2 & 2 & 2 & 2 & 1\end{array}\right.$ $\left.\begin{array}{ll}1 & 2\end{array}\right)$ Where, Sa represents Stride Length stream with tag character chosenis ' $a$ '. The traditional DFA for the rule *abba. $\{2\}$ caca is shown as follows:

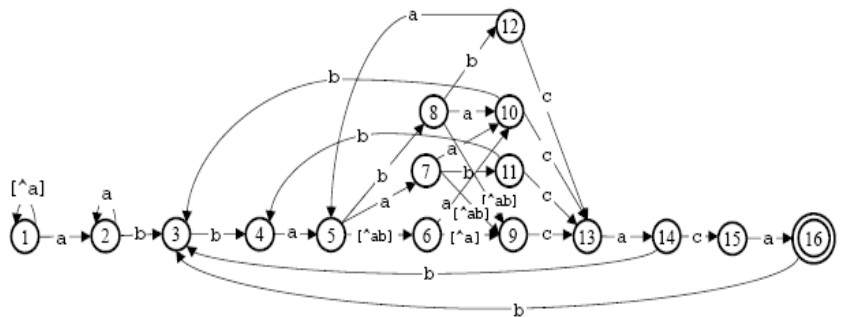

Fig. 3. Traditional DFA

Now the stride FA for the rule .*abba. $\{2\}$ caca is shown as follows:

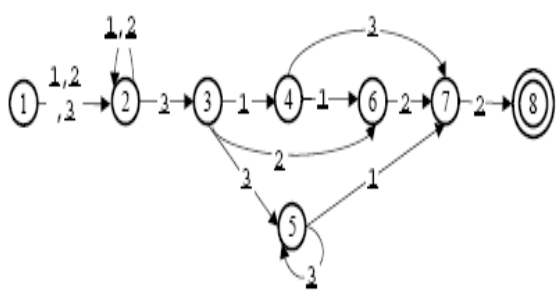

Fig.4. StrideFA

From now on, theinput characters arranged by the Deterministic Finite Automata has been streamlined. Therefore, the common DFA needs to analyze 17 data characters, however the proposed robot simply needs to channel 6 data walk lengths.However, the customary automata must be changed so as to affirm the entire number data stream (known as StriDFA). The standard and variety walk automatonfor the given data rule are showed up in Figs. 3 and 4, exclusively. Note that the advances in the StriDFA figure are set apart with SLs instead of characters The advancement of the StriDFA in this model is uncommonly direct. We first need to change the data to entire number walk streams. By then throughgeneral approach of creating DFA, make StriDFA.

Excellent change in the planning speed, perfect memory use, and straightforwardness of use on existing stages, are the upsides of walk automata.

\section{PROPOSED SCHEME}

The model for walk based robot contains three basic modules: walk length convertor, walk machine arranging motor, and string check module.

1. The walk length convertors change the pledge to different number walk length streams subject to different imprint characters.

2. walk robot arranging motor anticipate a key movement, which matches the responsibility against typical clarification rules, same as that of a conventional framework.

3. At last, through the confirmation sort out, a potential match is gotten by all walk automata. 


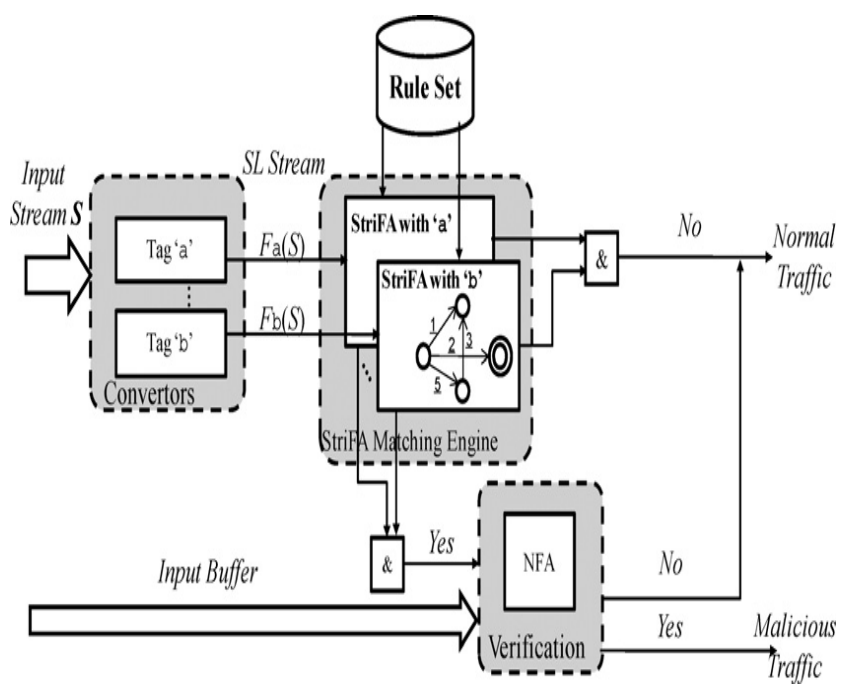

Fig .5.ArchitectureofStriFA-basedmatchingengine.

Stream layout addresses how to change over regexes to StriNFA/StriDFA

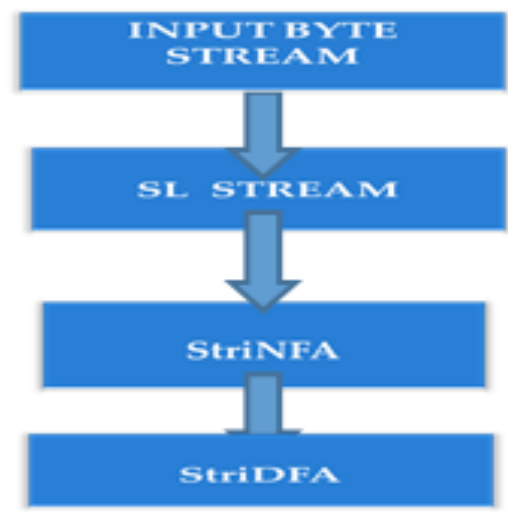

Fig.6. Flow Chart representing the designing of StrideFA

Source input character sequence will be converted to much stronger integer byte stream.

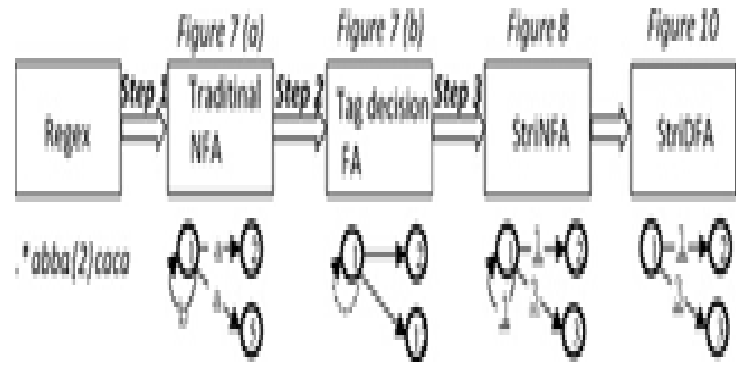

Fig.7.Convertion of regextoStriNFA/StriDFA

\section{A. Tag Selection}

It is definitely not hard to find that picking "visit" characters in a string as marks to streamline false positive rate.

\# of occasions of $\mathrm{c}$ in regex $\mathrm{r}$ over the aggregate of lengths of each and every fixed substring in $\mathrm{r}$ :

$$
\operatorname{Freq}(c, r)=\frac{\sum_{s \in \mathcal{S}_{c, r}}(\# c \text { in } s)}{\sum_{s \in \mathcal{S}_{c, r}}|s|}
$$

\section{B. Benefits of Stride FA:}

(a)Increased speed:
On the off chance that the lengths between imprints (fittingly picked) are passably long, the method can accomplish a tremendous brisk up.

(b) Small memory utilization:

The states consider is streamlined well as the selfconclusive gigantic limitless letter set is obliged by the window size.

\section{CONCLUSION}

In this paper, a novelregex model sorting out consider named walk robot is made to improve the show with nearness efficiencies. The key thought of walk automatontransforms the duty to various number walk length streams and a brief timeframe later apply it to a changed Deterministic Finite Automaton, called walk robot. The formal procedural estimation of walk robot, that rewritesrandom set of measures to a walk machine. We also portrayed the strategy to pass on walk length stream with the target that hoax positive can be reduced to an admirable level,

\section{REFERENCES}

1. K. Heyse, K. Bruneel, and D. Stroobandt, "Proving correct ness of regular expression matchers with constrained repetition," Electronics Letters, vol. 49, no. 1, pp. 41-42, 2013.

2. X. Wang, Y. Xu, O. Ormond, B. Liu, and X. Wang, "StriFA: stride finite automata for high-speed regularexpression matching in network intrusion detection systems," IEEE Systems Journal, vol. 7, no. 3 , pp. 374-384,2013.

3. H. Lu, K. Zheng, B. Liu, X. Zhang, and Y. Liu, "A memory-efficient parallel string matching architecture for high-speed intrusion detection," IEEE J. Sel. Areas Commun., vol. 24, no. 10, pp. 1793-1804, Oct. 2006.

4. S. Dharmapurikar and J. W. Lockwood, "Fast and scalable pattern matching for network intrusion detectionengines," IEEE J. Sel. Areas Commun., vol. 24 no. 10, pp. 1781-1792, Oct. 2006.

5. F. Yu, Z. Chen, Y. Diao, T. V. Lakshman, and R. H. Katz, "Fast and memory-efficient regular expression matching for deep packet inspection," in Proc. ACM/IEEE Symp. ANCS, Dec. 2006, pp. 93-102.

6. P. Hershey and C. Silio, "Surmounting data overflow problems in the collection of information for emerging high-speed network systems," IEEE Syst. J., vol. 4, no. 2, pp. 147-155, Jan. 2010.

7. M. Becchi and P. Crowley, "A hybrid finite automaton for practical deep packet inspection," in Proc. ACM Int CoNEXT, Dec. 2007, pp. 1.

8. M. Becchiand P. Crowley, "Efficient regula expression evaluation: Theory to practice," in Proc $4^{\text {th }}$ ACM/IEEE Symp. Architectures Netw.Commun. Syst., Nov. 2008, pp. 50-59.

9. R. Smith, C. Estan, S. Jha, and S. Kong, "Deflating the big bang: Fast and scalable deep packet inspection with extended finite automata," in Proc. ACM SIGCOMM, vol. 38, no. 4, pp. 207-218, Aug. 2008.

10. R. Smith, C. Estan, and S. Jha, "XFA: Faster signature matching with extended automata," in Proc. IEEE Symp.Security Privacy, May. pp.187-201 . 
11. Song, T., Zhang, W., Wang, D. and Xue, Y. (2008) A Memory Efficient Multiple Pattern Matching Architecture for Network Security. 27th IEEE Int. Conf. Computer Communications, Phoenix, AZ, USA, April 13-18, pp. 166-170.

12. Van Lunteren, J. (2006) High-performance PatternMatching for Intrusion Detection.25th IEEE Int. Conf.Com- puter Communications, Barcelona, Spain, April 23-29, pp. 1-13.

13. Becchi, M. and Crowley, P. (2007) An Improved Algorithm to Accelerate Regular Expression Evaluation. Proc. ACM/IEEE Symp. Architectures for Networking and Communications Systems, Orlando, FL, USA, December 3-4, pp. 145-154.

14. Tan, L. and Sherwood, T. (2005) A High Throughput String Matching Architecture for Intrusion Detection and Prevention.ISCA '05: Proc. 32nd Annual Int. Symp. Computer Architecture, Madison, WI, USA, June 4-8, pp.112-122.

15. S. Dharmapurikarand J. W. Lockwood, "Fast and scalable pattern matching for network intrusion detectionsystems," IEEE JSAC, vol. 24, no. 10, 2006.

16. Y. Fang, R. H. Katz, and T. V. Lakshman, "Gigabit rate packet pattern- matching using tcam," in IEEE ICNP,2004

17. M. Alicherry, M. Muthuprasanna, and V. Kumar, "High speed pattern matching for network ids/ips," in IEEEICNP, 2006.

18. D. P. Scarpazza, O. Villa, and F. Petrini, "Exact multipattern string matching on the cell/b.e. processor," in ACMCF, 2008.

19. C. R. Clark and D. E. Schimmel.Scalable pattern matching for high-speed networks.In IEEE FCCM, April2004.

20. B. Brodie, R., and D. Taylor. A scalable architecture for high-throughput regular-expression pattern matching. SIGARCH Comput.Archit. News, 34(2):191-202, 2006.

21. ReetinderSidhu,Vikot K. Prasanna "Fast Regular Expression Matching using FPGAs"9th Annual SymposiumIEEE2001.

\section{AUTHORS PROFILE}

Krishna Kishore Thota, Research Scholar, Computer Science Engineering,Sathyabama Institute of Science and Technology,Chennai,India. Email: thota.krishnakishore@gmail.com

Dr. R. JebersonRetna Raj, Associate Professor, Information Technology, Sathyabama Institute of Science and Technology, Chennai, Tamilnadu, India. Email: jebersonretnaraj.it@sathyabama.ac.in 